\title{
La agricultura campesina en Paraguay según las teorías clásicas sobre la cuestión agraria
}

\section{Peasant agriculture in Paraguay according to the classic theories on the agrarian issue}

\author{
Quintin Riquelme ${ }^{a}$
}

\begin{abstract}
Resumen
Esta investigación aborda una primera aproximación a las transformaciones del sector agrario paraguayo desde las tres tradiciones teóricas más importantes a partir de las cuales se ha analizado la cuestión agraria: la diferenciación campesina de Lenin; la organización de la unidad económica campesina de Chayanov y la descomposición campesina por la industria y el comercio de Kautsky. La realidad observada en el campo actualmente amerita mirarla desde esas tradiciones teóricas. El estudio es de carácter analítico, se revisan las teorías mencionadas cotejándolas preliminarmente con el proceso agrario paraguayo. El debate entre estas teorías no se ha agotado, al contrario, en momentos en que la agricultura moderna capitalista avanza, el problema en el campo se profundiza y se hace más necesaria concentrar la atención en ello. La complejidad de la realidad rural reflejo de la heterogeneidad del régimen de producción y de la estructura social, se traduce a su vez en la disparidad de las construcciones teóricas sobre el mismo fenómeno. Contrastadas éstas teorías con una aproximación a la realidad rural paraguaya no resulta fácil identificar cuáles de ellas reflejan con mayor precisión el actual proceso agrario. En Paraguay todavía persisten y conviven una diversidad de modalidades de estructuras productivas, las cuales están insertas de manera también diversa en la estructura productiva nacional y global.
\end{abstract}

Palabras clave: agricultura campesina, transformación agraria, teorías sobre la cuestión agraria.

\footnotetext{
Abstrac

This paper is an initial step in addressing transformations in the Paraguayan agrarian sector taking into account some of the most important traditional theories that have been used to analyze the agrarian question: Lenin on peasant differentiation; Chayonov on the organization of peasant economic unity, and Kautsky on peasant decline due to industry and commerce. The current reality, as observed in the field, merits a view from these theoretical traditions. The study is analytical, reviewing the above-mentioned theories
}

Kera Yvoty: reflexiones sobre la cuestión social. Vol. 1, 2016, 8o-9o.

ISSN (impreso): 2519-7797

a Universidad Nacional de Asunción, Instituto de Trabajo Social, Paraguay.

Correspondencia a: quintinriquelme@gmail.com

Cita:

Riquelme, Q. (2016). La

agricultura campesina en Paraguay según las teorías clásicas sobre la cuestión agraria. Kera Yvoty: reflexiones sobre la cuestión social, 1, 80-90.

Recibido:

13 octubre 2016

Aceptado:

29 noviembre 2016 
while making a preliminary comparison with the Paraguayan agrarian process. The debate among these theories has not been exhausted; on the contrary, in a moment in which modern capitalist agriculture is advancing, problems in rural areas are deepening thus necessitating greater attention to this issue. The complexity of the present situation in rural areas, a reflection of the heterogeneity of production regimes and social structures, in turn translates into a disparity in theoretical constructs about this same phenomenon. In contrasting these theories, along with a critical analysis of the reality in rural Paraguay, which theory more precisely reflects the current agrarian process is not easily identifiable. Diverse modes of structures of production still persist and exist side by side in Paraguay, as do the diverse ways in which they are inserted into national and global structures of production.

Keywords: peasant agriculture, agrarian transformation, theories on the agrarian question.

\section{Introducción}

La presente investigación aborda una primera aproximación a las transformaciones del sector agrario paraguayo desde finales de la década de 1970, a partir del proceso de modernización tecnológica de la agricultura y de los cambios en las políticas públicas dirigidas al sector. La nueva realidad observada -polarización entre minifundio-latifundio agroindustrial y ganadero, disminución de las pequeñas fincas y de la población campesina, continuidad delos conflictos sociales en tornoa la tenencia de la tierrayal agronegocio- amerita mirar el proceso agrario paraguayo, desde las tradiciones teóricas más importantes a partir de las cuales se ha analizado la cuestión agraria desde los inicios de estas transformaciones. Los debates más importantes e influyentes fueron propiciados por autores que desde sus distintas posiciones políticas e ideológicas analizaron el problema agrario y construyeron sus teorías apelando a rigurosos análisis de la documentación de la época y del proceso vivido por los protagonistas.

En Paraguay, las transformaciones agrarias más importantes comenzaron a producirse con la venta masiva de las tierras públicas a las grandes empresas agroforestales después de la guerra de 18651870 (Pastore, 1972), pero fue a partir de la década de 1970, con la modernización agraria que el capitalismo comenzó a expandirse en el campo, con lo cual, la agricultura campesina paulatinamente fue perdiendo su posición como principal actor productivo del país comenzando su crisis de la cual no se recupera hasta el presente (Riquelme \& Vera, 2016).

La concentración de la tierra por la agricultura empresarial y por la ganadería, por una parte, y el repliegue de la agricultura campesina, por otra, constituyen desde entonces dos problemas centrales del Paraguay, con consecuencias directas en el deterioro de vida de la población campesina, que finalmente conduce a la emigración. Los datos estadísticos oficiales proveídos por los censos agropecuarios realizados en diferentes momentos de la historia del país -1921, 1944, 1956, 1981, 1991 y 2008- confirman esta realidad (Gaona, 1987 y MAG 1956, 1981, 1991, 2008).

Cabe señalar que en Paraguay los dos modelos de explotación agropecuarios han existido; por una parte, el de la agricultura campesina cuya principal característica es la producción de rubros agrícolas diversificados destinados mayoritariamente al consumo; y, por otra, el de la agricultura empresarial cuya característica es la producción de rubros para la exportación y para la industria. Estos dos modelos durante varias décadas convivieron porque cada cual tenían delimitadas sus áreas de influencia sin afectarse mutuamente. A partirdelosaños 1970, con la modernización agraria, la incursión del capitalismo en el campo se vuelve más agresiva y mucho más con la aparición de la biotecnología hacia 
finales de la década de 1990 con sus semillas genéticamente modificadas y el paquete tecnológico que la acompaña.

Teniendo presente estas observaciones, el trabajo se propone recuperar el debate sobre las principales teorías de la cuestión agraria repensando la actualidad rural del país desde esas concepciones teóricas. Las tres propuestasa ser revisadas son: i) la diferenciación campesina desarrollada por Lenin en su libro El desarrollo del capitalismo en Rusia; ii) La organización de la unidad económica campesina de Alexander Chayanov; y iii) La Cuestión Agraria de Karl Kautsky.

Estos autores desde los últimos años del siglo XIX y principios del XX comenzaron aanalizarlos cambios operados en el sector agrario ruso y alemán y más específicamente en la economía campesina, formulando cada cual sus teorías frente a estos cambios y marcando tendencias que perduran hasta el presente. Cabe señalar que los planteamientos de Lenin y Kautsky se basan en las formulaciones originarias de Marx sobre la cuestión agraria.

El estudio es de carácter analítico, se revisan los textos de los tres autores mencionados centrándose en las principales propuestas teóricas de cada uno de ellos.

Las dos preguntas que guían esta investigación son:

- ¿Es relevante en la actual coyuntura del proceso agrario paraguayo repensar la cuestión agraria desde estas concepciones teóricas?; y

- ¿Cuáles de estas teorías son las más pertinentes para abordar la cuestión agraria en Paraguay?

El supuesto del cual se parte es el significativo cambio operado en el sector agrario paraguayo, el cual altera de manera visible el escenario rural. Este cambio es resultado, por un lado, de la implantación de un modelo de desarrollo que prioriza la producción a gran escala, debilitando el modelo de producción campesino basado en la diversidad productiva y en el uso sustentable de los recursos naturales y, por otro lado, la ausencia de una estrategia de desarrollo rural por parte del Estado que propicie la producción en pequeñas fincas. Los dos factores señalados colocan en el escenario la probabilidad de la desaparición paulatina de la agricultura campesina en gran parte del país, y mucho más en aquellas regiones donde el agronegocio es dominante.

\section{Marco referencial}

En el periodo en que surgen estas formulaciones teóricas, fines del siglo XIX y principios del XX, en Rusia se desarrollaba una fuerte polémica entre populistas y marxistas. El populismo sostenía que la base de una nueva organización social sería la comuna campesina. En este contexto surge la Escuela de Organización Producción de cuya fundación participa Chayanov. Esta Escuela proponía la transformación de la organización dela economía campesina para elevar la producción agrícola. En contraste, los marxistas proponían la nacionalización de la tierra primero y la socialización de la agricultura después (Bartra, 1987). Algunos populistas consideraban al campesinado como baluarte de la sociedad rusa por ser el depositario de los valores y las tradiciones y otros por sus potencialidades para instaurar un colectivismo socialista racional. La crítica marxista, especialmente de Lenin, consideraba al populismo como una ideología pequeñoburguesa que idealiza en extremo la vida económica de la comuna campesina (García Jurado, 2010).

Las tres principales tradiciones teóricas sobre la cuestión agraria.

\subsection{La diferenciación campesina}

Una de las teorías sobre la cuestión agraria es la diferenciación campesina desarrollada por Lenin en su libro El desarrollo del capitalismo en Rusia (1979), en el que plantea que el campesino es una categoría en transición cuya desaparición progresiva se dará en la medida que el capitalismo avanza en la agricultura. Este campesino, dice Lenin, se desdoblará en 
dos, una parte se convertirá en burguesía rural y otra en asalariado rural. A este proceso de diferenciación, le llama descampesinización, la desaparición paulatina del campesinado en aquellas regiones en las cuales el capitalismo agrario incursiona de manera más acelerada y cuya característica es la concentración y la exclusión.

Lenin (1979) llega a esta conclusión al revelar que el "medio económico-social en que se halla el campesinado ruso [...] es el de una economía mercantil. Incluso en la zona Central, la más atrasada [...] el campesino se halla totalmente supeditado al mercado" (p. 161). En consecuencia, en el régimen de las relaciones económicosociales del campesinado se observa todas las contradicciones características de la economía mercantil, -acaparamiento de tierra (comprada y tomada en arriendo), concentración de la producción en manos de una minoría y desplazamiento de la mayoría a las filas del proletariado. Con esta caracterización refuta la idea de que "el régimen de las relaciones económicas en la aldea de la comunidad sea un tipo económico especial -producción popularsostenida por los populistas, sino un tipo pequeñoburgués corriente" (p. 162).

Lenin (1979) dice:

No hay ni un solo fenómeno económico entre los campesinos que no tenga esa forma contradictoria, propiedad específica del régimen capitalista, es decir, que no exprese la lucha y la disparidad de intereses, que no represente un más para unos y menos para otros. Contrariamente a las teorías reinantes en nuestro país durante el último medio siglo, el campesino comunal ruso no es antagónico con respectoal capitalismo: es, al contrario, su base más profunda y más firme. (p. 162)

Afirma que a mayor avance de la economía mercantil, mayor será la separación de la población de la agricultura, es decir, el crecimiento de la población industrial es a costa de la agricultura. Citando a Marx, señala "por su naturaleza misma, el modo capitalista de producción hace disminuir constantemente la población agrícola con respecto a la no agrícola” (Lenin, p. 23).

Crítica los estudios realizados por los populistas sobre el tema de la diferenciación campesina por concluir éstos que la misma es resultado de un simple brote de las desigualdades en los bienes, como una simple diferenciación. Para Lenin (1979), la aparición de la desigualdad es el punto de partida que no termina en una simple diferenciación, sino que además de diferenciarse el "viejo campesinado se derrumba por completo, desplazado por tipos de población rural totalmente nuevos y que constituyen la base de la sociedad mercantil y de la producción capitalista" (p. 164).

De este viejo campesinado dice, surgen dos tipos de población rural, ambos con un rasgo común, su economía es de carácter mercantil y monetario. El primer tipo es la burguesía rural o los campesinos acomodados. De este grupo acomodado sale la clase de los farmers que es un tipo de productor que tiene capacidad de acumulación. Agranda su área de siembra arrendando tierras o comprándola a los pequeños. En este tipo de agricultura, el volumen de la producción supera a la fuerza de trabajo familiar, razón por la cual recurre a trabajadores externos.

El otro tipo nuevo es el proletariado rural, incluye a los campesinos pobres, a los sin tierras, pero los representantes típicos son el bracero, el jornalero, el peón, el obrero de la construcción o de otra clase con nadiel. Los campesinos con nadiel están en plena decadencia por la imposibilidad de subsistir sin vender su fuerza de trabajo. Tienen un nivel en extremo bajo, incluso inferior al obrero sin nadiel, "tales son los rasgos distintivos de este tipo de agricultores" (Lenin, p. 167).

Porotra parte, admitela lentitud con la 
que el capitalismo penetra en la agricultura por la diversidad de características del campesinado en los distintos países. "Cada uno de ellos ostenta las huellas de un régimen agrario peculiar, de una historia peculiar de relaciones agrarias" concluye (Lenin, p. 68).

\subsection{La organización de la unidad económica campesina}

Esta teoría, sostenida fundamentalmente por Alexander Chayanov, defiende la existencia de otro régimen de producción no asimilado dentro del marco de la teoría moderna de la economía, es decir la capitalista.

Chayanov (1987) expresa:

En la teoría moderna de la economía se ha hecho costumbre pensar todos los fenómenos económicos en relación exclusivamente con la economía capitalista -renta, precio, capital- Todos los demás tipos (no capitalistas) de vida económica se consideran insignificantes o en proceso de extinción [...] Por lo tanto no presentan interés teórico [...] En el pensamiento económico no podremos avanzar tan sólo con las categorías capitalistas, porque una región muy vasta de la vida económica (la parte más grande de la producción agraria) se basa no en una forma capitalista, sino en la forma completamente diferente de una unidad económica familiar no asalariada. (p. 49)

Afirma que en la mayoría de las explotaciones campesinas de los países menos desarrollados de Asia, África e incluso en algunos países europeos, ignoran las categorías de trabajo asalariado y salario, razón por la cual propone superar el marco conceptual de la economía moderna.

En una economía natural dice Chayanov (1987), la obligación es satisfacer las necesidades de cada unidad de producción, que es al mismo tiempo una unidad de consumo. Por lo tanto, el cálculo hay que hacer sobre la cuantía de la necesidad, por ello, el presupuesto es en alto grado cualitativo: "para cada necesidad familiar ha de proveerse en cada unidad económica el producto cualitativamente correspondiente in natura" (p. 52).

Esta teoría sostiene que una familia campesina logra satisfacer su necesidad cuando se produce un equilibrio entre la producción y el consumo. Cuando la producción es deficitaria con respecto al consumo, la familia campesina destina más tiempode trabajoa la producción hasta lograr de nuevo el equilibrio y viceversa, cuando hay abundancia de producción, se reduce el trabajo. Este equilibrio se logra de forma muy variable, dependiendo de las condiciones específicas reales de la producción de la unidad, su situación respecto al mercado y su ubicación. También importa el tamaño y la composición de la familia y la urgencia de sus necesidades. Cuando la productividad del trabajo es alta, se obtiene la misma cantidad de producto con menos trabajo, en cambio, en una economía campesina con varios miembros incapaces de trabajar, aumenta la sobreexplotación del trabajo para la satisfacción de las necesidades (Chayanov, p. 55).

"La cuantía del producto del trabajo dice Chayanov (1987), la determinan principalmente el tamaño y la composición de la familia trabajadora, el número de sus miembros capaces de trabajar y, además, la productividad de la unidad de trabajo y el grado del esfuerzo de los trabajadores, el grado de autoexplotación mediante el cual los miembros laborantes efectúan cierta cantidad de unidades de trabajo en el curso del año" (p. 54).

Ese grado de explotación lo determina un equilibrio peculiar entre satisfacción de la demanda familiar y fatiga del trabajo. Cuando no se alcanza la satisfacción de la demanda de consumo se trabajará más y viceversa, cuando se alcanza la cantidad suficiente para el consumo se dejará de trabajar, de autoexplotarse. 


\subsection{La descomposición campesina por la industria y el comercio}

Una tercera teoría surge a partir de la posición asumida por Karl Kautsky, en su libro La Cuestión Agraria, escrita en las primeras décadas del siglo pasado.

Kautsky (1989) afirma:

El modo de producción capitalista se desarrolla -excepción hecha de algunas colonias- fundamentalmente en las ciudades y en la industria. La agricultura permanece, por lo general, al margen de este proceso por mucho tiempo. Pero ya el mismo desarrollo industrial tiende a modificar el carácter de la producción agrícola. La familia campesina medieval constituía una comunidad económica que se bastaba a sí misma, autosuficiente; una comunidad que no solamente producía sus propios medios de subsistencia, sino que también construía su vivienda, sus muebles y utensilios domésticos, que fabricaba la mayor parte de sus elementales instrumentos de trabajo, curtía las pieles, hilaba el lino y la lana, confeccionaba sus ropas, etc. El campesino iba al mercado, por cierto, pero no vendía sino un sobrante de su producción, comprando aquello superfluo, excepción hecha del hierro, del que se servía muy limitadamente, de cómo le fuera en el mercado podía depender su comodidad y lujo, pero de ningún modo su existencia. (p. 1989)

Comparada esta situación con la de los actuales campesinos, dice Kautsky, habremos de convenir en que desde entonces se ha operado una gran revolución económica.

El primer paso en esta dirección ha sido la disolución del artesanado campesino por obra de la industria urbana y del comercio. Según Kautsky (1987), esa agricultura campesina autosuficiente, fue herida de muerte con el desarrollo de la industria urbana y correlativamente del comercio (p. 19). Cuanto mayor es el desarrollo del comercio así como de los medios de comunicación, mayor será la dependencia de la agricultura del comercio.

Si bien Kautsky señala que la agricultura fue herida de muerte por la industria y el comercio, afirma por otra parte, que el desarrollo de la gran explotación no es incompatible con el sostenimiento de la pequeña producción. Pero mostró que este sostenimiento no era por la superioridad productiva de la misma sino por el aporte de fuerza de trabajo en el territorio.

Citando a otro autor Kautsky (1987) señala: "el gran propietario territorial consigue los mayores beneficios, brutos y netos, cuando en torno a él hay una legión de pequeños y medianos propietarios que le abastecen de fuerza de trabajo y adquieren el excedente de sus productos" (p. 175). De esto deduce "que no hay que suponer que la explotación en pequeña propiedad agraria se encuentre a punto de desaparecer en la sociedad moderna y deba ser completamente sustituida por la gran propiedad" (p. 195).

Tampoco la pequeña propiedad se mantendrá porque es capaz de competir con la grande. Subsiste justamente porque deja de ser competitiva a la grande y porque produce la mercancía que la gran hacienda necesita, la mercancía fuerza de trabajo (Kaustky, p. 195). "Cuando las cosas han llegado a este punto, la gran hacienda y la pequeña no se excluyen, sino que se condicionan, al igual que el capitalista y el proletario, pero el pequeño agricultor asume aquí de un modo creciente la condición de proletario" (Kaustky, p. 196).

\section{El proceso agrario paraguayo en la perspectiva de las teorías planteadas}

El debate entre estas teorías no se ha agotado, al contrario, en momentos en que la agricultura moderna capitalista avanza, el problema se profundiza y se hace más necesaria concentrar la atención en ello. 
En Paraguay en aquellos departamentos en los cuales el avance del capitalismo agrario es más intenso, la diferenciación del campesinado también es mucho más acelerada. En Departamentos como Alto Paraná, Canindeyu, Itapúa, Caaguazú, las grandes parcelas aumentan a costa de las pequeñas. Alto Paraná de 1.489. $500 \mathrm{Ha}$, de superficie total, en el período agrícola 2013/14 la superficie sembrada de soja llegó a 987 ooo Ha, en Canindeyú a 666.30o Ha de una superficie total de 1.446.70o Ha. Caaguazú, la extensión total de soja fue de $455.184 \mathrm{Ha}$ de una superficie total de 1.147.400 Ha e Itapúa a 629.900 Ha de un total de 1652.500 Ha (MAG, 2015). En estos departamentos la teoría leninista de la diferenciación campesina se cumple con relativa certeza. Los agricultores de pequeñas fincas van desapareciendo progresivamente, por la venta de sus tierras, por el arriendo y por el desahucio.

En los lugares donde este capitalismo avanza más lentamente, como en los Departamentos de Paraguarí, Guairá, es probable que la descampesinización, en su sentido estricto -desaparición o disminución de las pequeñas fincas campesinas- tarde más en producirse, pero, los efectos igualmente tienen profundas consecuencias sobre los modos de vida campesinos. El capitalismo permea todo, las sociedades, las comunidades, las familias, generando nuevas necesidades muchas de las cuales son creadas artificialmente, pero produce gran impacto en los modos de vida, en la cultura de la población. Al no poder satisfacer las demandas que la sociedad de consumo le impone con el ingreso de su finca, el campesino busca satisfacer esas demandas desde fuera de sus fincas, produciéndose el desarraigo.

Cuando en Paraguay se alude a la diferenciación campesina, quizás sea importante abordarla no sólo desde la razón de la desaparición o disminución paulatina de las pequeñas fincas, sino también desde la destrucción de sus modos de vida que tienen que ver con su producción, con su forma de alimentación, con su identidad y con su forma de relacionarse con el entorno social y ambiental.

En razón de estas realidades heterogéneas, es posible afirmar que se da una diferenciación relativa. No se puede negar la descampesinización tanto en su dimensión cuantitativa como cualitativa en Paraguay, tampoco se puede afirmar que la misma se da con la misma intensidad y magnitud en todos los lugares, lo cual lleva a la necesidad de profundizar la investigación, apelando a la documentación secundaria y a los datos primarios de investigación de campo.

El planteamiento de Chayanov de analizar el régimen de la producción campesina desde fuera de las categorías económicas del capitalismo -plusvalía, renta, salario-, presentándolo como una unidad económica autosuficiente merece igualmente una atención especial. La agricultura campesina autosuficiente, inmersa en su propia lógica de producción y de consumo, en su organización productiva autónoma es probable que sea inviable en las condiciones actuales del desarrollo productivo, pero tampoco se puede confirmar su desaparición. Es necesario continuar indagando para develar su existencia o no. Chayanov reconoce la existencia de más de un tipo de estructuras productivas en el mismo seno de la economía campesina, pero él se planteaba analizar sólo la morfología de la unidad económica doméstica familiar, su objetivo no era realizar el análisis de la unidad campesina en su relación con la economía nacional ni con la global, sino simplemente su funcionamiento interno.

El aporte de Chayanov desde ese punto de vista no deja de ser importante porque devela el funcionamiento de la estructura interna de la economía campesina y la necesidad del equilibrio entre producción y consumo. Pero este equilibrio puede no darse porque las unidades económicas campesinas no son homogéneas, en la producción intervienen varios factores. Él no oculta 
esta diferenciación, admite que una unidad productiva con escasa tierra, no todos los miembros de esa unidad podrán dedicarse a las tareas agrícolas en sus fincas, tendrán que buscar trabajo fuera de ellas para poder satisfacer la demanda de la economía familiar. También reconoce que aquellas unidades familiares con escasos miembros y con tierra de mala calidad, destinará mayor cantidad de trabajo para la satisfacción de las necesidades y la contratación de fuerza de trabajo será necesaria.

El dilema que se plantea es ¿podrá una unidad económica campesina autosostenerse sólo con lo que produce en la finca sin recurrir al mercado, sin vender su fuerza de trabajoy sin utilizar fuerza de trabajo externa? En el caso de Paraguay esta posibilidad es poco probable. La agricultura campesina está inmersa en la lógica del mercado, no importa cuál sea la cuantía de su producción y mucho más en la actualidad en la cual la producción extra agraria inunda el mercado y las comunidades rurales están expuestas a ella.

En una familia campesina muy pobre, necesariamente sus miembros deben destinar parte de su tiempo a trabajos extraprediales, de lo contrario no podrá satisfacer sus necesidades básicas incluso de alimentación. Sin embargo, en las familias sin necesidades extremas, es probable que sus miembros no necesiten vender su fuerza de trabajo y que trabajen sólo en sus fincas, pero es poco probable que no utilicen fuerza de trabajo externa en el proceso de producción. Las familias acomodadas con una superficie de tierra superior a la media, es seguro que recurran a la fuerza de trabajo externa, por la cuantía de su producción, por lo tanto la categoría salario está presente en el proceso de producción.

Kaustky por su parte asume una posición intermedia en el sentido de que ambos modos de producción se necesitan mutuamente. El modo de producción campesino como proveedora de fuerza de trabajo a la gran explotación y ésta como generadora de empleo y proveedora de insumos para las industrias.
Si Kaustky afirma que la compatibilidad y sostenibilidad entre ambas agriculturas se da porque la agricultura campesina es proveedora de fuerza de trabajo a la gran explotación, la pregunta obligada es ¿es sostenible este supuesto frente al avance tecnológico en la agricultura para el cual la fuerza de trabajo manual dejó de ser necesaria? Este supuesto debería ser revisado con mayor detenimiento, desde el momento en que la gran explotación está totalmente tecnologizada (maquinarias, semillas transgénicas, usos de agroquímicos) y en la cual la fuerza de trabajo manual dejó de ser un componente necesario. La biotecnología ha convertido la fuerza de trabajo manual en superflua.

En los inicios de la modernización agraria, las teorías sobre la economía campesina sostenían la teoría de Kaustky, la funcionalidad de la pequeña explotación campesina a la agricultura empresarial moderna por la provisión de fuerza de trabajo. En el caso de Paraguay, han habido experiencias en las cuales las grandes empresas agroindustriales (caso Agriex en Alto Paraná 1980) destinaban parte de sus tierras a la creación de asentamientos campesinos para tener mano de obra barata y disponible'. Pero esa funcionalidad desde un tiempo a esta parte dejó de ser tal, las máquinas y la bioteconología han desplazado a la mano de obra.

Finalmente, Kautsky (1989) sostiene: es la gran industria capitalista la que domina y la agricultura debe seguir su marcha, adaptarse a sus necesidades. La dirección del desarrollo industrial regula el desarrollo agrícola [...] Pero en las zonas que permanecen puramente agrícolas, sea por inaccesibilidad a su territorio, sea por

\footnotetext{
1 El caso fue observado en ocasión de la realización de un estudio sobre la migración brasileña en los departamentos de Alto Paraná y Canindeyú, propiciado por el Comité de Iglesias para Ayudas de Emergencia (CIPAE) año 1980.
} 
el carácter cerrado de sus habitantes, todavía permanecen cerradas a la penetración de la industria, la población decae desde el punto de vista del número de la fuerza, de la inteligencia, del nivel de vida, y con ella se empobrece el suelo, la hacienda agrícola decae. La agricultura pura y simple no constituye más, en la sociedad capitalista, un elemento de bienestar. Al mismo tiempo desaparece también la posibilidad de una renovada prosperidad del sector campesino. (p. 354)

En esta cita, Kautsky (1989) desarrolla la idea de que el campesinado o la agricultura en pequeñas fincas permanecerá en la medida de su relacionamiento o vinculación con la industria y con el mercado. "La agricultura pura y simple no constituye más, en la sociedad capitalista, un elemento de bienestar" sostiene.

En Paraguay, este proceso se observa de alguna manera en la región central del país, en los departamentos de Central, Cordillera, Paraguarí y parte de Guairá, en los cuales, una gran proporción de la población campesina ya no se dedica con exclusividad a la agricultura, sino se inserta en el mercado de trabajo urbano, en la industria, el comercio y los servicios, sin abandonar sus antiguas comunidades. En éstas siguen produciendo los rubros agrícolas necesarios para el complemento de la canasta básica.

\section{Conclusión}

La complejidad de la realidad rural, reflejo de la heterogeneidad del régimen de producción y de la estructura social, se traduceenladisparidaddelasconstrucciones teóricas sobre el mismo fenómeno. Los tres autores parten de presupuestos teórico-políticos y metodológicos distintos que los llevan a conclusiones también distintas. Contrastadas éstas teorías con una aproximación a la realidad rural paraguaya no resulta fácil identificar cuáles de ellas reflejan con mayor precisión el actual proceso agrario, en el que todavía persisten y conviven una diversidad de modalidades de estructuras productivas, las cuales están insertas de manera también diversas en la estructura productiva nacional y global.

No hay duda de que la diferenciación y la descomposición campesina se producen en Paraguay y con mayor velocidad en aquellas áreas donde el capitalismo agrario incursiona de manera más agresiva, pero el mismo fenómeno también se observa en áreas no dominadas por la gran explotación. No por casualidad Paraguay perdió $38 \mathrm{mil}$ fincas en sólo 6años, del 2002 al 2008, (MAG, 2002 \& 2008) pero ¿cuánto habrá perdido desde el 2008?, es una de las preguntas que necesitan ser respondidas. Pero que no se va a responder si es que no se dispone de un censo o de una encuesta representativa. Sobre la evolución de la cantidad de fincas y su extensión y la PEA rural ver texto sobre la cuestión agraria en libro de FAO/ OIT/CEPAL de 2012 con artículo de J M A Verdecchia y ver también en capítulo sobre Trabajo Decente y Desarrollo Humano en el área rural, informe de PNUD con OIT de 2013.

Otra pregunta que no puede dejarse de plantear es: ¿Qué pasó con las 38 mil pequeñas fincas desaparecidas y cuántos de los dueños/as de esas fincas pasaron a formar parte de la burguesía rural y cuántos del proletariado rural? Los datos empíricos disponibles no permiten realizar inferencias de ese tipo, pero, es posible suponer que la mayoría de las fincas pasó a ampliar las fincas mayores. Por otra parte, por la escasa industrialización de la economía paraguaya, tampoco se puede afirmar que la fuerza de trabajo liberada en la agricultura se traduzca en un trabajador asalariado dependiente. En la mayoría de los casos esas fuerzas laborales pasan a formar parte de los trabajadores informales cuentapropistas en la periferia de las ciudades.

Paraguay sigue siendo uno de 
los países del Mercosur con la más alta ruralidad, el $40 \%$ de su población vive en áreas rurales. Se ha comentado que este enfoque es una aproximación porque faltan elementos empíricos relevantes; en la PEA rural de 2015, el sector primario es $46 \%$, el secundario es $17 \%$ y el resto es terciario $37 \%$. Aún en 2010, el sector primario de la PEA rural es $60 \%$ y el secundario $13 \%$ y el resto es terciario. Este $60 \%$ del 2010 está muy lejos del 93\% calificados como campesinos en un año no especificado. Esto supone la presencia de por lo menos tres tipos de estructuras productivas, pequeñas, medianas y grandes. Cada cual a su vez desarrollan diferentes tipos de relaciones de producción. La primera por lo limitado de su capacidad productiva debido a la poca disponibilidad de tierra, es probable que mantenga una escasa relación con el mercado, produce sólo lo que va a consumir y su aporte al mercado es casi nulo. En estos casos tampoco resulta fácil medir cuánto de lo que consume produce en su finca y cuánto de lo que produce lo hace sólo con la fuerza de trabajo familiar, es probable que no utilice fuerza de trabajo externa, pero lo que no podrá evitar es ofrecer su fuerza de trabajo fuera de su finca para satisfacer las necesidades básicas familiares.

Las medianas, en cambio probablemente mantengan una relación más fluida con el mercado sea vendiendo el producto de su trabajo, producido por la fuerza de trabajo familiar o por la fuerza de trabajo externa. Pero tampoco se puede asegurar con certeza que no sufran la pérdida de las fincas por presión de la gran explotación. Los datos del censo muestran que la mayor pérdida de fincas se produjo en las parcelas medianas de entre 5 a 20 hectáreas.

Las más grandes por su parte $(20$ a $50 \mathrm{Ha}$ ), por la cuantía de su producción se puede suponer que mantiene fuertes relaciones mercantiles y su producción ya no es sólo para el consumo familiar sino una proporción importante dirigida al mercado. Produce mercancías con valor de cambio, por lo tanto, se puede suponer que gran parte de la fuerza de trabajo utilizada en la producción sea externa. También es de suponer que la resistencia a la diferenciación sea menor, por su mayor capacidad productiva, de consumo y de ingreso, aunque la comparación de los censos agropecuarios 1991 y 2008 muestra una disminución también importante de este estrato de productores.

Otra pregunta que tampoco puede dejar de formularse. ¿Es posible sostener la existencia de un campesino autosuficiente deacuerdoacomoplanteaba Chayanov? Con los datos estadísticos disponibles y algunas observaciones preliminares realizadas, no es posible asegurar la existencia de un campesinado autosuficiente, inmerso en su lógica de producción. En el actual proceso agrario, la producción extra-agraria ha invadido el mercado, del cual el campesino, incluso el más pobre no está exento.

Finalmente, parecería que la sobrevivencia del campesinado dependerá de su relación con el mercado y con la industria. Un campesino que produce para su consumo y para el mercado de tal manera a generar ingresos monetarios para enfrentar los desafíos que le impone la sociedad de consumo. Pero esta probabilidad dependerá de las condiciones generadas por el Estado. Un Estado ausente para el sector campesino y con fuerte presencia para el agronegocio no revitalizará la economía campesina.

En resumen, las preguntas planteadas sobre la pertinencia de recurrir a estas tradiciones teóricas para repensar la cuestión agraria en Paraguay siguen siendo relevantes. Primero por la complejidad y profundidad de los cambios en el sector rural y segundo, por la necesidad de seguir buscando explicaciones y alternativas a un sector importante del país que corre el riesgo de ser desplazado totalmente del escenario rural si no operan cambios sustantivos en las políticas del Estado.

Esta inicial exploración de las transformaciones del proceso agrario paraguayo desde las perspectivas de las teorías analizadas, sugiere la necesidad de 
seguir profundizando la investigación dada la mayor complejidad del problema en el campo y por la imposibilidad de comprobar los supuestos que plantean cada una de las teorías, sin una información combinada de datos primarios y secundarios.

\section{Referencias Bibliográficas}

Bartra, R. (1976). Introducción a Chayanov. Nueva Antropología, 1(3), 49-69. Recuperado de http://www.redalyc. org/pdf/159/15900303.pdf

Chayanov, A. (1987). La organización de la unidad económica campesina. Segunda Edición. México: Siglo XXI Editores.

Gaona, F. (1987). Introducción a la historia gremial y social del Paraguay. Asunción: RP Ediciones.

García Jurado, R. (2010). Las raíces del populismo. Los movimientos populistas del siglo XIX en Rusia y Estados Unidos. Argumentos, 23(63), 267-288. Recuperado de http://www. scielo.org. $\mathrm{mx} /$ scielo.php?script $=$ sci_ arttext\&pid=So187-57952010000200011

Lenin, V. (1979). El desarrollo del capitalismo en Rusia. Moscú: Edit. Progreso.

Kautsky, K. (1989). La cuestión agraria. México, Siglo XXI Editores.
Ministerio de Agricultura y Ganadería. (1952). Censo Agropecuario Nacional 1951. Asunción: MAG.

Ministerio de Agricultura y Ganadería. (1982). Censo Agropecuario Nacional 1981. Asunción: MAG.

Ministerio de Agricultura y Ganadería. (1992). Censo Agropecuario Nacional 1991. Asunción: MAG.

Ministerio de Agricultura y Ganadería. (2002). Encuesta Agropecuaria 2002. Asunción: MAG.

Ministerio de Agricultura y Ganadería. (2008). Censo Agropecuario Nacional 2008. Asunción: MAG.

Ministerio de Agricultura y Ganadería. (2015). Síntesis Estadísticas. Producción agropecuaria. Año agrícola 2013/2014. Asunción: MAG.

Pastore, C. (1972). La Lucha por la tierra en el Paraguay. Montevideo: Ed. Antequera.

Riquelme, Q. \& Vera, E. (2016). Estudio sobre actualización de aspectos legales de la AFC en Paraguay. Asunción: CDE, DECIDAMOS. 